\title{
Performance enhancement of nonlinear asymmetric bistable energy harvesting from harmonic, random and human motion excitations
}

\author{
Wei Wang ${ }^{1}$, Junyi Cao ${ }^{1, a)}$, Chris R. Bowen ${ }^{2}$, Daniel J. Inman ${ }^{3}$, Jing Lin ${ }^{1}$
}

${ }^{1}$ State Key Laboratory for Manufacturing System Engineering, Xi'an Jiaotong University, Xi'an, 710049, China

${ }^{2}$ Materials and Structures Centre, Department of Mechanical Engineering, University of Bath, Bath, BA27AY,

UK ${ }^{3}$ Department of Aerospace Engineering, University of Michigan, Ann Arbor, Michigan 48109-2140, USA

\begin{abstract}
Numerical and experimental investigations of nonlinear bistable energy harvesters (BEHs) with asymmetric potential functions are presented under various excitations for performance enhancement. Basin of attraction under harmonic excitation indicates that asymmetric potentials in BEHs have negative effect on the power output. Therefore, a proper bias angle is introduced to the asymmetric potential BEHs for performance enhancement. Numerical and experimental results show that the power output is actually improved in a certain bias angle range under harmonic and random excitations. Furthermore, experiments under human motion excitation demonstrate that the asymmetric potential BEHs could perfectly combine with the asymmetric motion of lower-limb to improve the performance.
\end{abstract}

Keyword: Energy harvesting, Bistable, Asymmetric potential, Performance enhancement.

Due to the high sensitivity to low intensity excitations, bistable structures have received significant attention from a variety of research areas which include nonlinear energy harvesting ${ }^{1-4}$, weak signal detections $^{5,6}$, and optomechanical devices ${ }^{7}$. Particularly, vibrational energy harvesting is a field that witnessed the most extensive application of bistable configurations. Cottone et al. ${ }^{8}$ investigated the dynamic response of a bistable piezoelectric inverted pendulum subjected to Gaussian random excitations. Erturk et al. ${ }^{9,}{ }^{10}$ reported a bistable piezomagnetoelastic device for piezoelectric energy harvesting, and presented investigations into the high-energy branches (HEBs) of the bistable system over a range of excitation frequencies. Litak et al. ${ }^{11}$ numerically considered the stochastic characteristics of a BEH driven by stationary Gaussian white noise. Additionally, Daqaq ${ }^{12}$ investigated the response of a $\mathrm{BEH}$ subjected to Gaussian excitations and demonstrated that the shape of the optimal potential function is sensitive to the noise intensity. Zhao and Erturk ${ }^{13}$ focused on the relative advantages of bistable and monostable harvesters under random excitations, revealing that $\mathrm{BEH}$ can be preferred only if it is carefully designed to operate at a known excitation intensity. Furthermore, Zhou et al. ${ }^{14}$ proposed a BEH with two rotatable external magnets and demonstrated that improved performance was achieved compared with linear one. Although numerous efforts have been devoted to model and analyse the bistable structures for broadband energy harvesting, the majority of existing researches focus on bistable systems with symmetric potential energy functions.

However, it is very difficult or even impossible to fabricate a system with a completely symmetric potential function in practice due to imperfections in the bistable structures and material properties. Halvorsen ${ }^{15}$ investigated an asymmetric quartic potential bistable system and illustrated that asymmetric potentials have a significant influence on the output performance. He and Daqaq ${ }^{16}$ studied an asymmetric quadratic nonlinearity similar to Halvorsen ${ }^{15}$ and demonstrated that it appeared to degrade the system performance especially for low to moderate white noise intensity. Although, a detailed understanding of the influence of asymmetric potentials and corresponding enhancement strategies to improve harvested power from vibrations are still unexplored for nonlinear BEHs, especially when applied for practical cases with asymmetric excitations, just like human lower-limb motion $^{17}$. 
Therefore, numerical and experimental investigations will be carried out to focus on the performance enhancement of asymmetric potential BEHs under different excitations. When a BEH is applied to harvest energy from human motion shown in Fig. 1(a) ${ }^{18}$, the lower-limb will make the harvester swing over a certain angle (Fig. 1(b)). In this case, the dimensionless model of the BEH can be obtained as ${ }^{17}$

$$
\left\{\begin{array}{l}
\ddot{x}+2 \xi \dot{x}-x+\beta x^{2}+\delta x^{3}-p \sin \phi-\kappa^{2} V=f \cos (\Omega t), \\
\dot{V}+\alpha V+\dot{x}=0,
\end{array}\right.
$$

where $\xi$ is the damping ratio, $f$ is the excitation level and $\Omega$ represents the dimensionless frequency. $\delta$ is the cubic nonlinearity coefficient of restoring force and $\beta$ is the quadratic nonlinearity coefficient introduced to characterize the asymmetric potential of bistable configuration. $p$ is the equivalent gravity and $\phi$ is the bias angle. $\kappa^{2}$ is the dimensionless electromechanical coupling coefficient and $\alpha$ is the ratio between mechanical and electrical time constants. Also, $x$ and $V$ are respectively the displacement and voltage response. For the case of a symmetric potential with $\beta=0$ and $p=0$, the oscillator has the same probability ${ }^{19}$ to vibrate in each well directly or after transient chaos when it has insufficient energy to cross the potential barrier. Thus, oscillation from each well results in almost the same final fate and has no influence on the output. However, if a small asymmetry is introduced, for example with $\beta=1 / 12$, the oscillator has larger probability ( $81.6 \%$ ) to vibrate in the deeper potential well, as shown in Fig. 1(c). This has negative effect on the efficiency of the harvester both in simulation and experiments. In addition, this probability will rapidly increase and approach $100 \%$ as the asymmetry further increases.

(a)
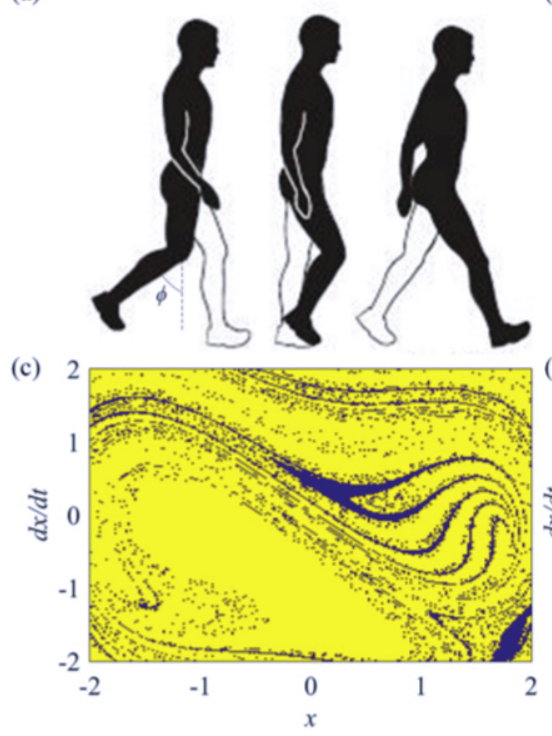

(b)

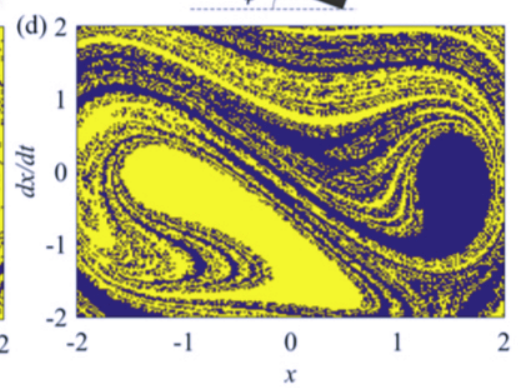

FIG. 1. (a) Posture of human lower-limb during walking ${ }^{18}$. (b) BEH with a certain bias angle. (c) Basin of attraction for a BEH with $\beta=1 / 12$ and $p=0$. (d) Basin of attraction when an asymmetric BEH is balanced. Yellow represents the oscillator with a final fate in the deeper potential well and dark for those in shallower one.

As a result of the change of system structural parameters or excitation condition in Eq. (1), e.g. during walking in Fig. 1(a), there is an optimum bias angle that balances the asymmetry induced by the quadratic nonlinearity. The optimal value $\phi$ is equal to $\sin ^{-1}\left\lceil\left(2 \beta^{3}+9 \beta \delta\right) 27 / p / \delta^{2}\right\rceil$ and it is opt $\rfloor$ about $10.3^{\circ}$ for the parameters used herein with $\beta=0.2199$. For the optimum bias angle, the basin of attraction given in Fig. 1(d) shows an approximately equal probability into each well and is close to 
the attraction basin for Eq.(1) with $\beta=0$ and $p=0$. Therefore, the bias angle could have a positive effect on the performance of the nonlinear $\mathrm{BEH}$ that exhibits asymmetric potentials.

Simulated data for the mean square value of voltage response $E\left[V^{2}\right]$ under sweep frequency excitations for a variety of bias angles are shown in Fig. 2(a). $E\left[V^{2}\right]$ of the system for different bias angles under up-sweep frequency excitation with an excitation of $f=0.5$ increases slowly with an increase of the bias angle and reaches the largest value approximately $10^{\circ}$ and then decreases as the bias angle further increases. In other words, the bandwidth for $\mathrm{HEB}^{20-22}$ is largest around the optimum bias angle and it becomes narrower when away from that. For down-sweep frequency excitation, the range of optimum bias angle for a larger power output is wider than that for up-sweep excitation, showing that the asymmetric potential has less impact on down-sweep than that for up-sweep frequency excitation.
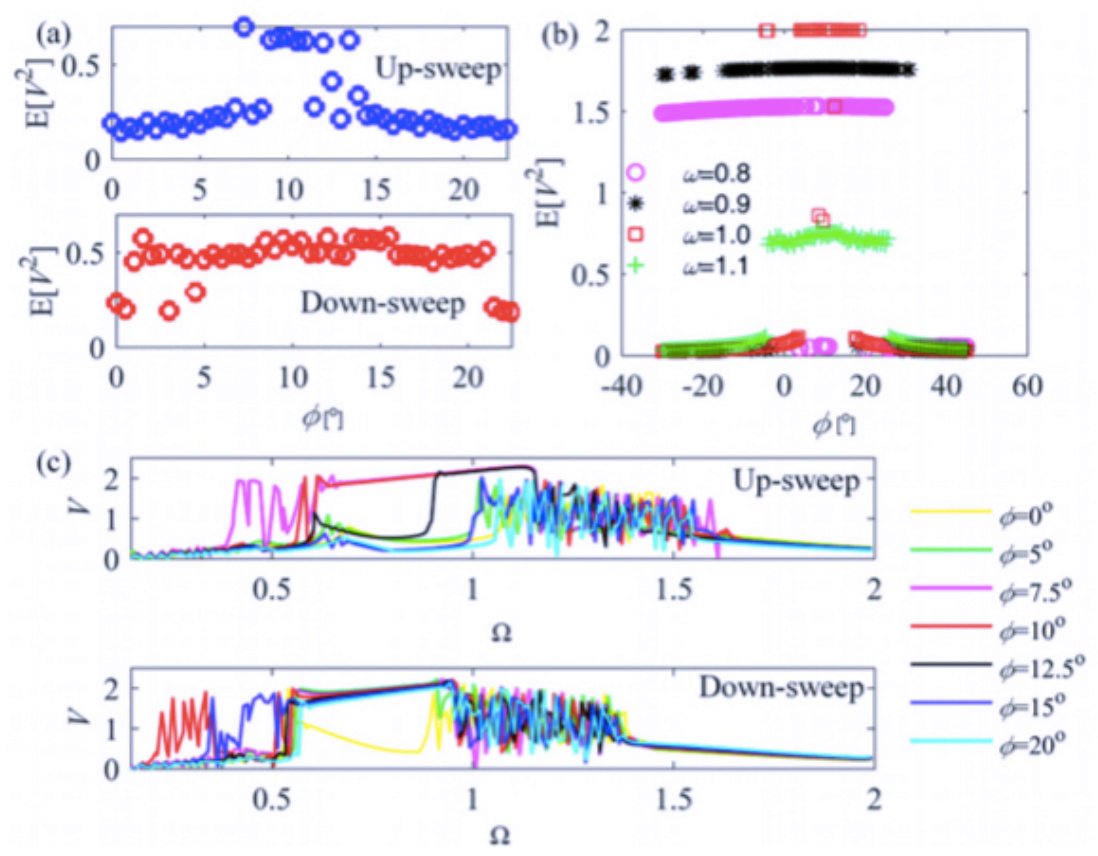

FIG. 2 Numerical simulation. (a) $E\left[V^{2}\right]$ of the system $(\beta=0.2199)$ under different bias for up and down-sweep frequency excitation with $f=0.5$. (b) $E\left[V^{2}\right]$ under different bias for various $\Omega$ with $f=0.5$. (c) Voltage response for up and down-sweep frequency excitation with level of 0.5 under several specific bias angles.

For detailed explanation, the voltage responses under up and down-sweep frequency excitation for several specific bias angles are shown in Fig. 2(c). At bias angles of 7.5o and 10o, the responses under up-sweep frequency excitation exhibit better performance than that at other bias angles. While for down-sweep frequency excitation, the harvester could experience the periodic inter-well motion at more bias angles except for 00 . For constant frequency excitations, the relationship between $E\left[V^{2}\right]$ and bias angle is shown in Fig. 2(b). In addition to the influence of frequency on the output, the range of bias angles for high-energy output also depends on the frequency. The optimum range of bias angle becomes narrower around the optimum angle for the frequencies from 0.8 to 1.0 which result in periodic inter-well motion. While, the oscillation in the optimum bias angle range for frequency of 1.1 is chaotic response. What's more, it is noted that there are some scattered points on low-energy branch in the optimum bias angle range, which may be due to the sensitivity of BEH to initial condition in the simulation. Additionally, the probability of surfing the HEB distinctly enlarges in the case of initially oscillating from a shallow potential well. This is why the optimum range on the left of optimum value is wider than on right side. 
(a)
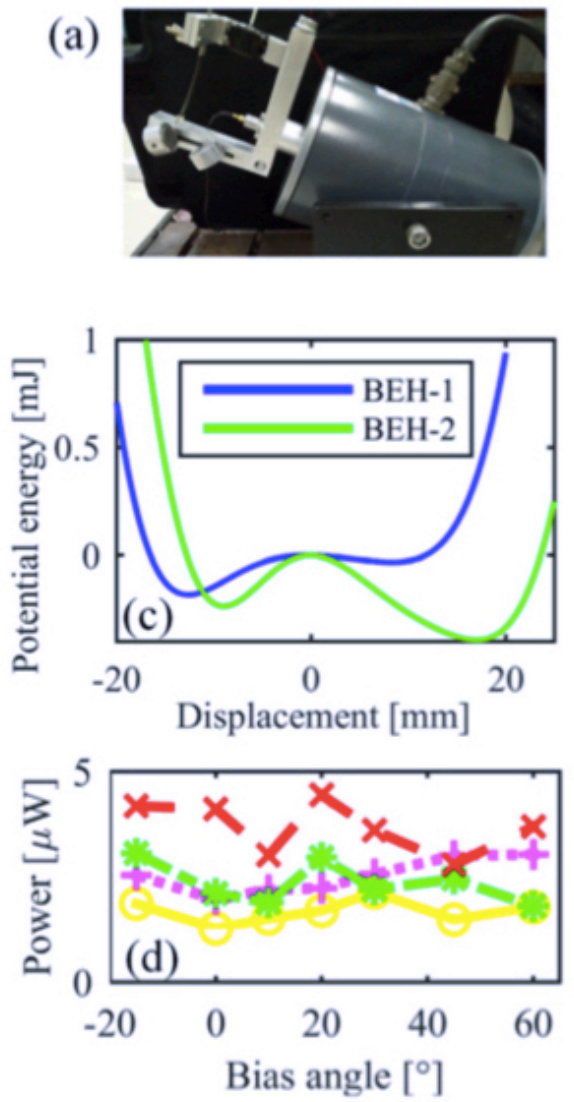

(b)

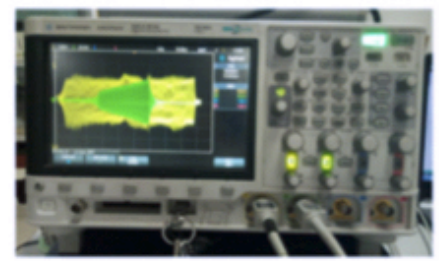

$-15^{\circ}-0^{\circ}-10^{\circ}-20^{\circ}$
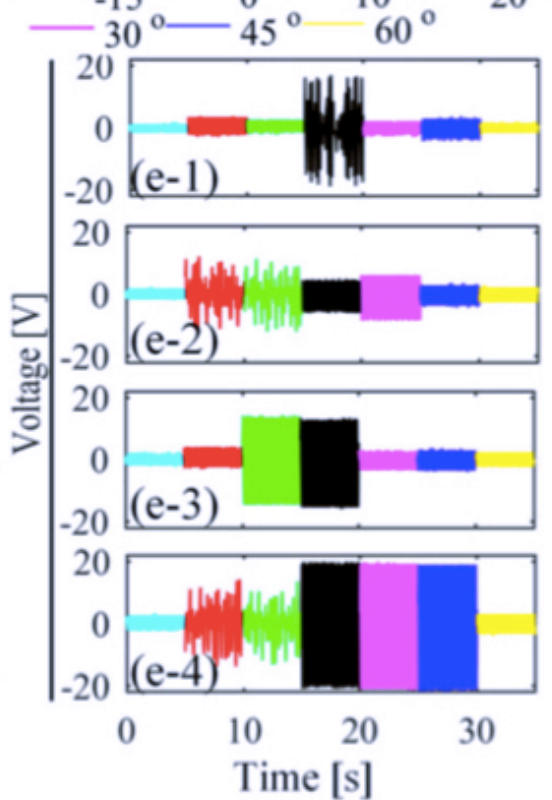

FIG. 3 (a) Experiment setup. (b) Oscilloscope for data collection (c) Potentials of asymmetric BEH-1 and BEH2. (d) Average output power of BEH-1 under random excitations at different bias angles: . (e) Voltage output of BEH-1 at different bias angles under constant frequency: (1) $0.3 \mathrm{~g}, 5 \mathrm{~Hz}$; (2) $0.3 \mathrm{~g}, 8 \mathrm{~Hz}$; (3) $0.4 \mathrm{~g}, 5 \mathrm{~Hz}$; (4) $0.4 \mathrm{~g}$, $8 \mathrm{~Hz}$.

The experimentally measured output of an asymmetric potential BEH shown in Fig. 3(a) is recorded by an oscilloscope MSOX3052A in Fig. 3(b). The potential energy functions of two asymmetric BEHs are plotted in Fig. 3(c), and BEH-1 ( $d=33 \mathrm{~mm}, h=17 \mathrm{~mm}, \vartheta=40 \mathrm{o})$ with shallower asymmetric potential wells compared to BEH-2 $(d=45 \mathrm{~mm}, h=21 \mathrm{~mm}, \vartheta=0 \mathrm{o})$ is initially discussed for experimental illustration under harmonic and random excitation. In the experiments, the responses of BEH- 1 are investigated at several bias angles of $-15^{\circ}, 0^{\circ}, 10^{\circ}, 20^{\circ}, 30^{\circ}, 45^{\circ}$ and $60^{\circ}$. When the bias angles are $0^{\circ}, 10^{\circ}$ and $20^{\circ}$, BEH-1 still keeps a bistable configuration, while it shows monostable property for other bias angles. Fig. 3(d) shows the average output power of BEH-1 under band-limited random excitations with different levels and frequency ranges. When the harvester exhibits a monostable configuration in the deeper potential well with a bias angle of $-15^{\circ}$, the output power under any excitation is improved, while a monostable configuration in the shallower well will degrade the performance. Due to bias angle balancing of the asymmetric potential, the output increases with the bias angle increasing from $0^{\circ}$ to $20^{\circ}$. In addition, an increase of excitation intensity and the extension of excitation frequency range could improve the performance of the harvester and the maximum average output power in the experiments is $4.46 \mu \mathrm{W}$. 

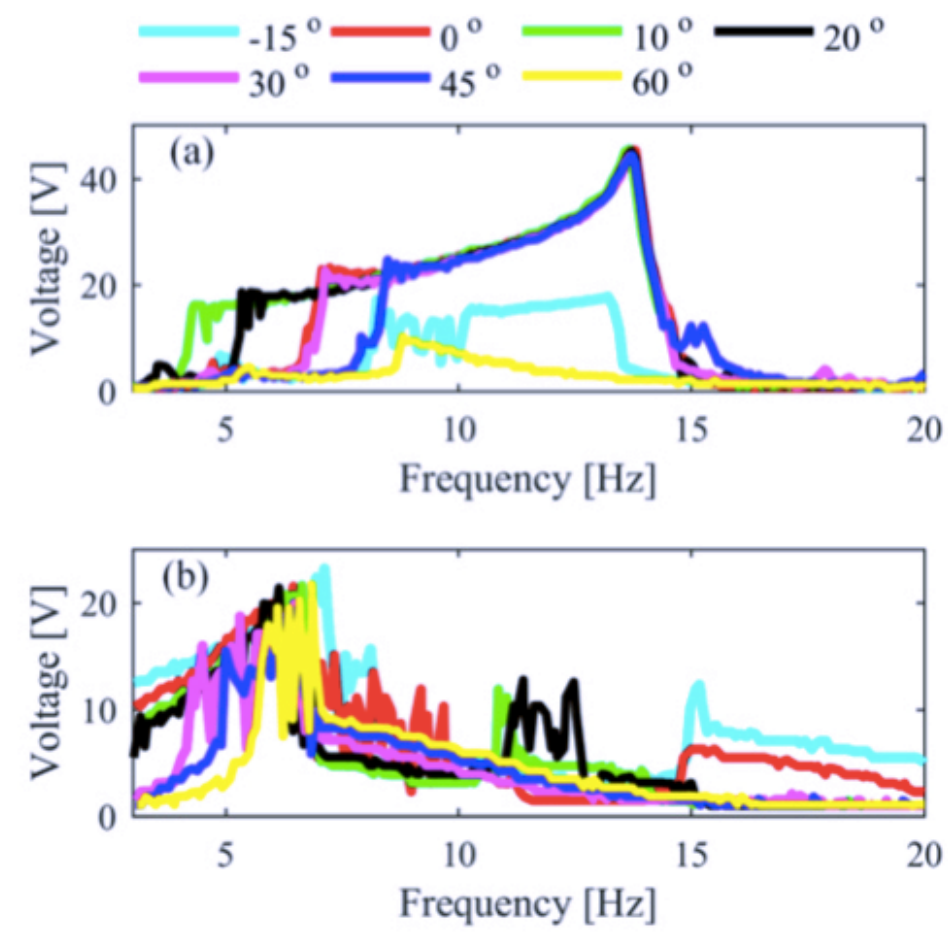

FIG. 4 Voltage response of BEH-1 under up (a) and down (b) sweep frequency excitation with level of 0.4g.

Under harmonic excitation with acceleration of $0.3 \mathrm{~g}$ and frequency of $5 \mathrm{~Hz}$ (Fig. 3(e-1)), the output of BEH-1 firstly increases and then decreases with an increase in the value of bias angle, with the maximum output power at a bias of $20^{\circ}$ exhibiting a chaotic response. When the frequency is increased to $8 \mathrm{~Hz}$ (Fig. 3(e-2)), the BEH-1 exhibits chaotic dynamics for bias angles of $0^{\circ}$ and $10^{\circ}$, while small amplitude intra-well motion for other values. Under excitation of $0.4 \mathrm{~g}$ and $5 \mathrm{~Hz}$ (Fig. 3(e3)), BEH-1 obtains the HEB for bias of $10^{\circ}$ and $20^{\circ}$, while it is on the low-energy branch for other values of bias angle. However for a larger frequency $8 \mathrm{~Hz}$ (Fig. 3(e-4)), in addition to the chaotic response at bias of $0^{\circ}$ and $10^{\circ}$, the optimum bias for HEB increases to $20^{\circ}, 30^{\circ}$ and $45^{\circ}$. Fig. 4(a) shows the response of BEH-1 under up-sweep frequency excitation of $0.4 \mathrm{~g}$, it is viewed that the frequency range for BEH-1 on HEB firstly becomes wider and then narrower with the increasing of

the bias angle. The optimum frequency range is from $4.2 \mathrm{~Hz}$ to $14 \mathrm{~Hz}$ at a bias of $10^{\circ}$. For downsweep frequency excitation (Fig. 4(b)), the frequency range for HEB shifts to higher frequencies with the increase of the bias angle due to the change in potential energy functions. The agreement between experimental results and numerical simulations demonstrates that balancing the asymmetric potential with a proper bias angle could enhance the performance of an asymmetric potential BEH. 

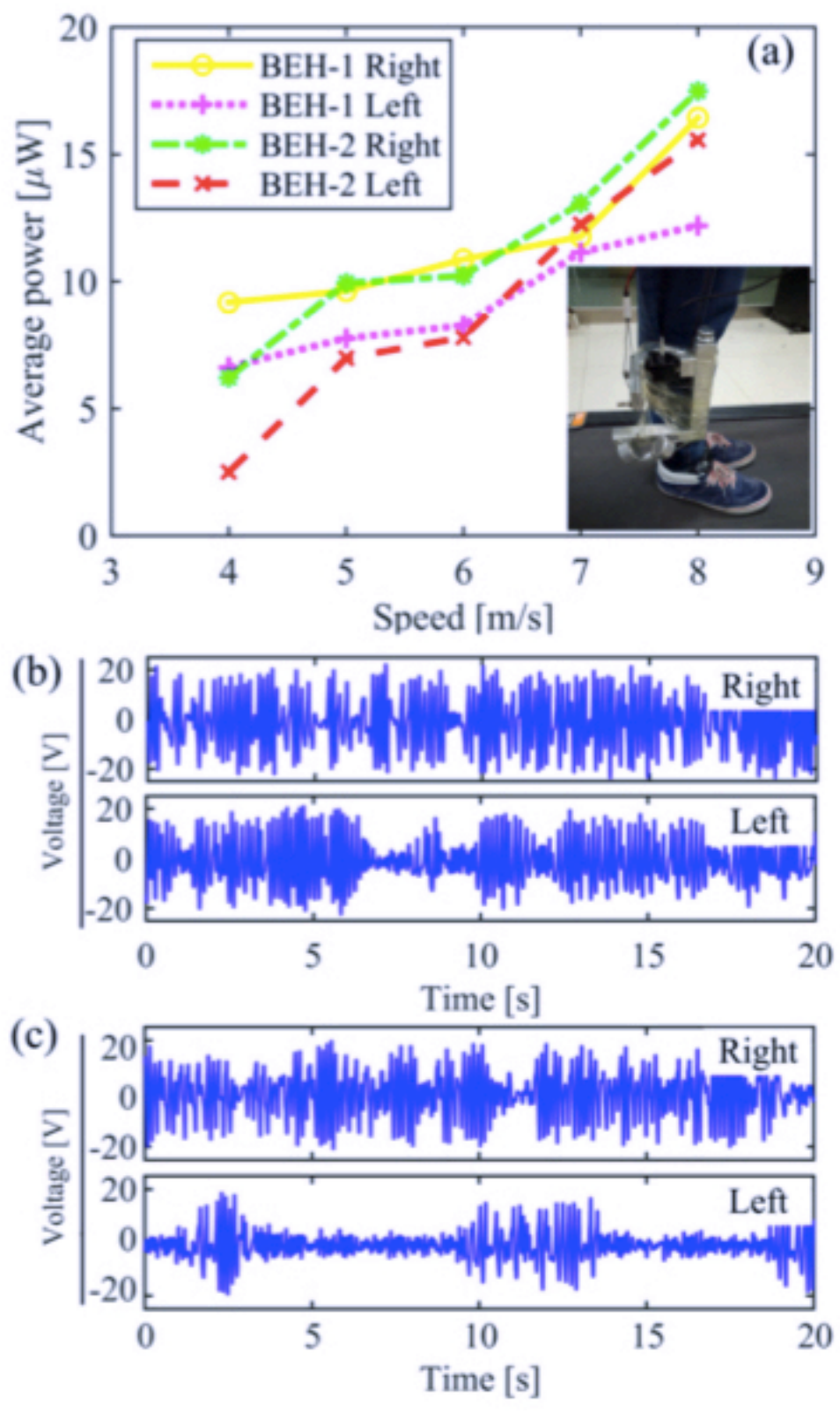

FIG. 5 Experimental results under human motion excitation. (a). Average output power of BEH-1 and BEH-2 under various motion speeds, also experiment setup is shown; Voltage response of BEH-1 (b) and BEH-2 (c) under speed of $4 \mathrm{~km} / \mathrm{h}$ for right and left leg.

For human motion excitation, a human participant with weight of $60 \mathrm{~kg}$ and height of $175 \mathrm{~cm}$ undertook walking or running at a speed of $4 \sim 8 \mathrm{~km} / \mathrm{h}$ on a treadmill to record the output. Fig. 5(a) shows the average output power of BEH-1 and BEH-2 at different motion speeds, along with the experiment setup on a treadmill. When the asymmetric BEH is attached to right leg, the deeper potential well is behind and the shallower one is forward, which can be perfectly balanced for the reason that human leg swings a larger angle to backward asymmetrically ${ }^{17}$. However, the influence is opposite for the left leg. For each harvester studied, the output power increases with the increase of motion speed and the maximum output power obtained in the experiments is $16.43 \mu \mathrm{W}$ for BEH-1 and $17.47 \mu \mathrm{W}$ for BEH-2. Due to the balance between potentials and asymmetric lower-limb motion, $\mathrm{BEH}$ on the right leg generates a larger output power than that on left leg. The influence of this mechanism is more evident at lower motion speed for a harvester with deep potential wells (BEH-2). For a clear description, the voltage responses of BEH-1 and BEH-2 on right and left leg at speed of 4 $\mathrm{km} / \mathrm{h}$ are shown in Fig. 5(b) and Fig. 5(c) respectively. For BEH-1 with shallower potential wells on 
right and left leg, the oscillator all can readily travel across the potential wells and the influence mechanism is less obvious. However for BEH-2 with deeper potential wells, the oscillator can jump the potential wells frequently for the right leg with output power of $6.22 \mu \mathrm{W}$, while the performance greatly decreases for that on left leg which only generate power with $2.51 \mu \mathrm{W}$.

In summary, numerical and experimental investigations into asymmetric potential issue in bistable energy harvesting system are provided for performance enhancement. Due the negative effect of asymmetric potentials on the power output, a proper bias angle of the system is proposed to enhance the performance. Numerical simulations under constant and sweep frequency excitations indicate that there is an optimal bias angle range for enhancing the performance of the asymmetric $\mathrm{BEH}$, which is also demonstrated by the experimental results. What's more, the output powers under random excitation with different bandwidth and intensity show a trend of growth due the bias angle in a certain range. Further, experiments under human motion excitation validate that the asymmetric potential BEHs could perfectly combined with the asymmetric motion of human lower-limb to enhance the performance and a maximum power of $17.47 \mu \mathrm{W}$ is obtained under motion speed of 8 $\mathrm{km} / \mathrm{h}$.

Support from National Natural Science Foundation of China (Grant Nos. 51575426, 51611530547 and 51421004), Novel Energy Materials, Engineering Science and Integrated Systems (NEMESIS) (Grant No. 320963), and Fundamental Research Funds for the Central Universities of China (Grant No. xjj2016002); this research is partly supported by China Scholarship Council.

1. ${ }^{1}$ M. F. Daqaq, R. Masana, A. Erturk, and D. Dane Quinn, Appl. Mech. Rev. 66, 040801 (2014).

2. $\quad{ }^{2}$ R. L. Harne and K. W. Wang, Smart Mater. Struct. 22, 023001 (2013).

3. ${ }^{3}$ S. P. Pellegrini, N. Tolou, M. Schenk, and J. L. Herder, J. Intell. Mater. Syst. Struct. 24, 1303 (2012).

4. ${ }^{4}$ L. Tang, Y. Yang and C. K. Soh, J. Intell. Mater. Syst. Struct., 21, 1867 (2010).

5. ${ }^{5}$ H. Chen and P. K. Varshney, IEEE T Signal Proces. 56, 5031 (2008).

6. ${ }^{6}$ H. Chen, P. K. Varshney, S. M. Kay, and J. H. Michels, IEEE T Signal Proces. 55, 3172 (2007).

7. 7 F. Monifi, J. Zhang, Ş. K. Özdemir, B. Peng, Y. X. Liu, F. Bo, F. Nori, and L. Yang, Nature Photon. 10, 399 (2016).

8. ${ }^{8}$ F. Cottone and H. Vocca, L. Gammaitoni. Phys. Rev. Lett. 102, 080601 (2009).

9. ${ }^{9}$ A. Erturk, J. Hoffmann, and D. J. Inman, Appl. Phys. Lett. 94, 254102 (2009).

10. ${ }^{10}$ A. Erturk and D. J. Inman., J. Sound Vib., 330, 2339 (2011).

11. ${ }^{11}$ G. Litak, M. I. Friswell and S. Adhikarl, Appl. Phys. Lett., 96,214103 (2010).

12. ${ }^{12}$ M. F. Daqaq, Nonlinear Dynam., 69,1063 (2012).

13. ${ }^{13}$ S. Zhao and A. Erturk, Appl. Phys. Lett., 102,103902 (2013).

14. ${ }^{14}$ S. Zhou, J. Cao, A. Erturk and J. Lin, Appl. Phys. Lett., 102,173901 (2013).

15. ${ }^{15}$ E. Halvorsen, Phys. Rev. E 87, 042129 (2013).

16. ${ }^{16}$ Q. F. He and M. F. Daqaq, J. Sound Vib. 333(15), 3479-3489 (2014).

17. ${ }^{17}$ J. Cao, W. Wang, S. Zhou, D. J. Inman, and J. Lin, Appl. Phys. Lett. 107,143904 (2015).

18. ${ }^{18}$ J. M. Donelan, Q. Li, V. Naing, J. A. Hoffer, D. J. Weber, and A. D. Kuo, Science. 319, 807 (2008).

19. ${ }^{19}$ S. H. Strogatz, Nonlinear dynamics and chaos( Westview press, 2014).

20. ${ }^{20}$ S. Zhou, J. Cao, D. J. Inman, S. Liu, W. Wang, and J. Lin, Appl. Phys. Lett. 106, 093901 (2015).

21. ${ }^{21}$ D. Mallick, A. Amann, and S. Roy, Phys. Rev. Lett. 117, 197701 (2016).

22. ${ }^{22}$ S. Zhou, J. Cao and J. Lin, Nonlinear Dynam., 86,1599 (2016). 